\title{
Analysis of progression of cervical OPLL using computerized tomography: typical sign of maturation of OPLL mass
}

\author{
Byung-Wan Choi, MD, ${ }^{1}$ Dong-Hoon Baek, MD, ${ }^{1}$ Lindsey C. Sheffler, MD, ${ }^{2}$ and Han Chang, MD ${ }^{3}$ \\ ${ }^{1}$ Department of Orthopedic Surgery, Inje University, Haeundae Paik Hospital, and ${ }^{3}$ Department of Orthopedic Surgery, Busan \\ Korea Hospital, Busan, Korea; and '2Department of Orthopedic Surgery, University of California, San Francisco, California
}

\begin{abstract}
OBJECT The progression of cervical ossification of the posterior longitudinal ligament (OPLL) can lead to increase in the size of the OPLL mass and aggravation of neurological symptoms. In the present study, the authors aimed to analyze the progression of cervical OPLL by using CT imaging, elucidate the morphology of OPLL masses, and evaluate the factors associated with the progression of cervical OPLL.
\end{abstract}

METHODS Sixty patients with cervical OPLL were included. All underwent an initial CT examination and had at least 24 months' follow-up with CT. The mean duration of follow-up was 29.6 months. Fourteen patients (Group A) had CT evidence of OPLL progression, and 46 (Group B) did not show evidence of progression on CT. The 2 groups were compared with respect to the following variables: sex, age, number of involved segments, type of OPLL, and treatment methods. The CT findings, such as the connection of an OPLL mass with the vertebral body and formation of trabeculation in the mass, were evaluated.

RESULTS Sex and treatment modality were not associated with OPLL progression. The mean age of the patients in Group A was significantly lower than that in Group B $(p=0.03)$. The mean number of involved segments was 5.3 in Group A and 3.6 in Group B ( $p=0.002)$. Group A had a higher proportion of cases with the mixed type of OPLL, whereas Group B had a higher proportion of cases with the segmental type $(p=0.02)$. A connection between the vertebral body and OPLL mass and trabeculation formation were more common in Group $B(p<0.01)$.

CONCLUSIONS Progression of cervical OPLL is associated with younger age, involvement of multiple levels, and mixed-type morphology. OPLL masses that are contiguous with the vertebral body and have trabecular formation are useful findings for identifying masses that are less likely to progress.

http://thejns.org/doi/abs/10.3171/2015.1.SPINE131167

KEY WORDS cervical; OPLL; progression; CT; trabeculation

$\mathrm{O}$ SSIFICATION of the posterior longitudinal ligament (OPLL) involves abnormal calcification of the posterior longitudinal ligament and mostly affects the cervical spine. Compression of the spinal cord by an OPLL mass can lead to neurological symptoms, and in cases of severe neurological deficits, surgery is required. The optimal method for treating asymptomatic or mildly symptomatic patients remains controversial because the exact pathogenesis and natural history of OPLL remain unclear. Studies on the progression of OPLL report that the size of the OPLL mass can increase, which can lead to aggravation of neurological symptoms in some patients, even after laminoplasty. ${ }^{6,8}$ Most studies have evaluated outcomes only in patients with OPLL who have undergone surgical intervention. Such data have limitations in predicting the prognosis or disease progression, especially in patients who are not treated surgically. Evaluating the progression of OPLL masses in operated and nonoperated cases using the same protocol can be more informative. Although plain radiography is the simplest method for detecting OPLL, both inter- and intraobserver reliability have been reported to be low, and this modality has limita- 
tions in detecting the early stages of the disease. ${ }^{1}$ To our knowledge, CT imaging has not been used for evaluating the progression of OPLL in any previous study that has included patients who were managed conservatively. Thus, in the present study, we aimed to analyze the progression of cervical OPLL using CT, report on the morphology of OPLL masses, and evaluate the factors associated with mass progression.

\section{Methods}

We retrospectively reviewed 104 consecutive cases of cervical OPLL managed at our institution between March 2008 and August 2012. The study was approved by the institutional review board of Haeundae Paik Hospital. Sixty subjects who were diagnosed with OPLL and had been followed up for $>24$ months were included in the analysis. The inclusion criteria were as follows: 1) diagnosis of cervical OPLL and 2) initial CT scan and subsequent follow-up CT scans using the same protocol, with the latest CT scan at least 24 months after the first (i.e., duration of imaging follow-up $\geq 24$ months). The exclusion criteria were loss to follow-up, history of trauma, and CT scan performed with a different protocol. The mean duration of followup was $29.6 \pm 4.6$ months (range $24-62$ months). The CT images (obtained with a Discovery CT750 HD scanner; GE Healthcare) of the disk spaces had a thickness of 1 $\mathrm{mm}$, whereas the vertebral body and sagittal planes had a thickness of $2 \mathrm{~mm}$. Based on the Investigation Committee on OPLL of the Japanese Ministry of Public Health and Welfare's classification, the OPLL types were categorized as local, segmental, continuous, and mixed. ${ }^{12}$

The progression of the OPLL mass on the CT studies was compared between the initial CT scan and the CT scan performed at the latest follow-up evaluation. The criteria for the progression of the OPLL mass were based on the following findings: 1) the mass progressed $>2 \mathrm{~mm}$ horizontally in the thickest area from the vertebral body to the OPLL mass; or 2) it progressed $>2 \mathrm{~mm}$ longitudinally based on the longest distance between the proximal and distal margins of the OPLL mass on the sagittal plane (Fig. 1) ${ }^{7}$

Patients were divided into 2 groups based on whether or not progression was evident at the most recent followup. Patients in Group A ( $\mathrm{n}=14)$ showed progression; patients in Group B $(n=46)$ did not. The CT data for all the participants were analyzed by 2 orthopedic spine surgeons based on the number of involved segments, types of ossification, and progression of the OPLL mass. The following variables were evaluated: sex, age, number of involved segments, history of operative treatment, and type of surgical intervention. The radiological findings of connection of the OPLL mass with the vertebral body and the formation of trabeculation in the OPLL mass were evaluated in the axial view (Fig. 2). All the CT measurements were performed using the institution's PACS (m-view, MaroTech). Two blinded observers who were orthopedic spine surgeons independently interpreted the radiological findings and performed the measurements, and the average findings were used in the analysis. When there was a difference in interpretation, the observers came to a mutual conclusion, which was used in the analysis. To verify
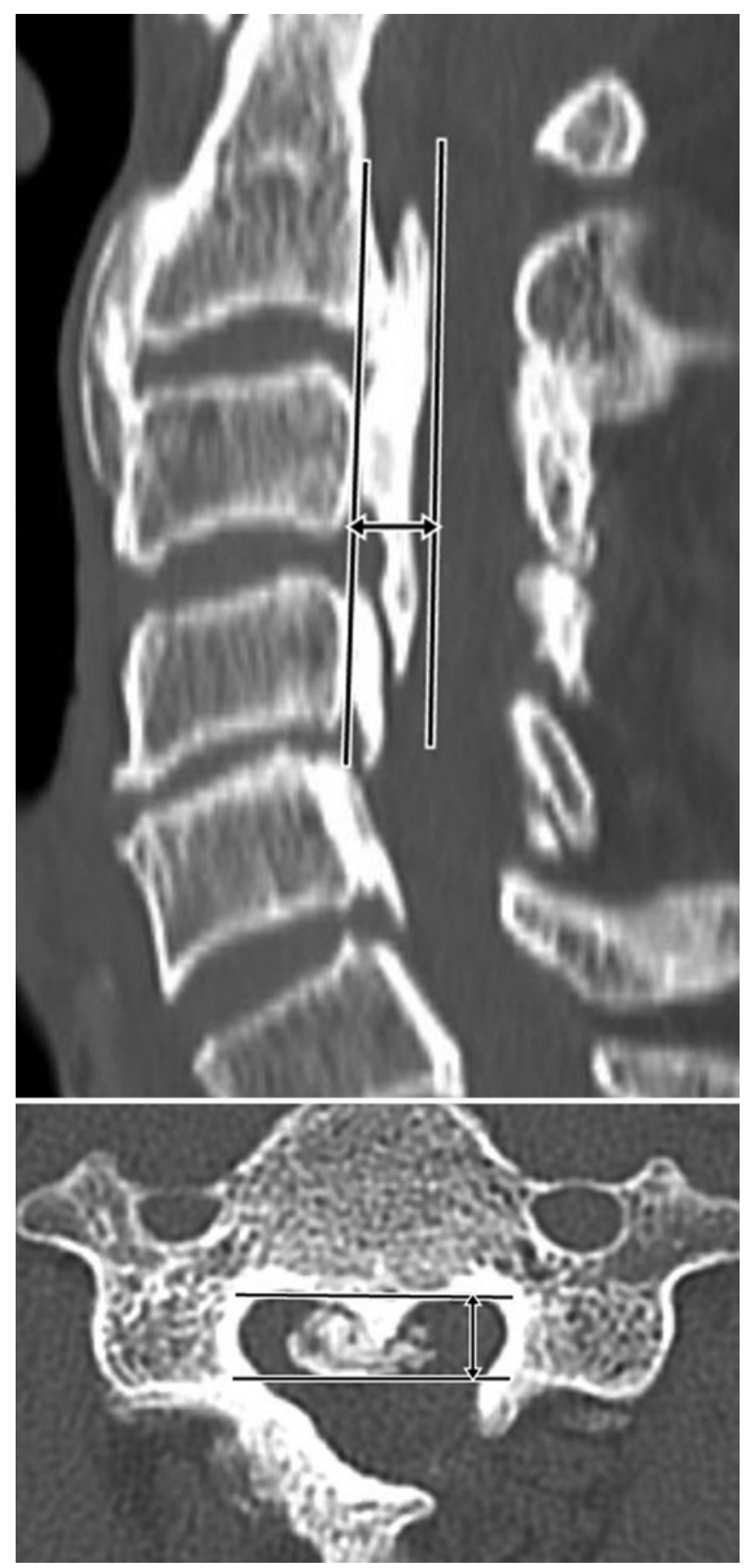

FIG. 1. Sagittal reconstruction and axial CT image illustrating the method for measuring the OPLL mass. The mass was measured longitudinally (upper) and horizontally (lower). The measurement was performed by assessing the distance (in the thickest area) from the posterior vertebral body to the OPLL mass.

the reliability of the measured values, the intra- and interobserver agreement was assessed using the kappa coefficient.

A sample size calculation for the primary analysis was conducted using $\mathrm{G}^{*}$ Power 3.1 (University of Kiel) with an anticipated effect size of Cohen's d $=0.8$, significance $[$ alpha $]=0.05$, and an expected power of $(1-[$ beta $])=0.8$. 


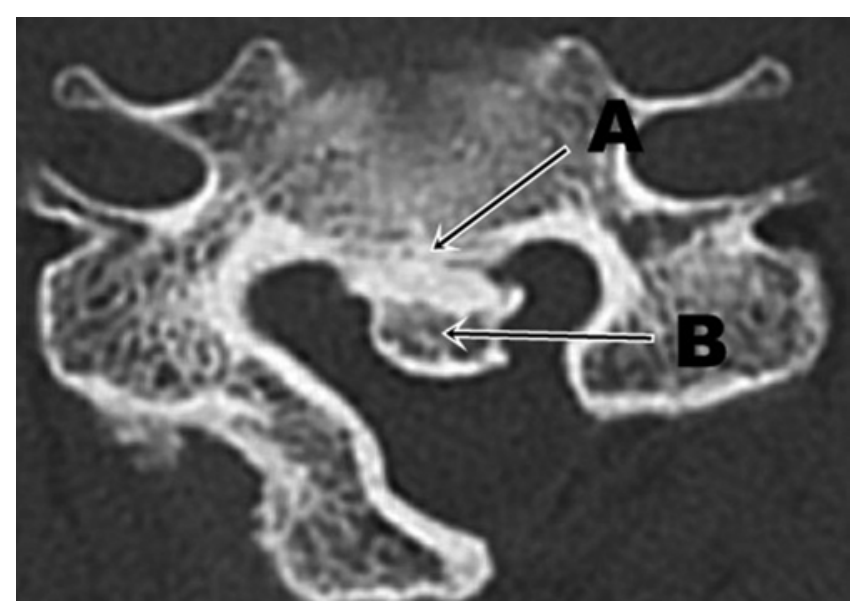

FIG. 2. Axial CT image demonstrating connection with the vertebral body (arrow $A$ ) and trabeculation (arrow $B$ ). This OPLL mass shows trabeculation, which is similar in appearance to the cancellous bone in the vertebral body, and it is clearly connected to the vertebral body.

This yielded a sample size of $\mathrm{n}=26$, which was determined using a 2-tailed distribution calculation.

SPSS (version 18.0, SPSS, Inc.) was used to perform the statistical analyses, and the differences between the groups were analyzed using the Mann-Whitney U-test and Fisher exact test. Lastly, multivariate linear regression analysis was performed to determine the factors associated with the progression of OPLL (the dependent variable). The results were deemed statistically significant at $\mathrm{p}<0.05$.

\section{Results}

Forty-eight men and 12 women were included in the analysis. The mean age of the overall group of 60 patients was $54.5 \pm 8.47$ years (range 29-72 years). There were no significant differences between the patients who showed progression of the OPLL at the latest follow-up (Group A, $\mathrm{n}=14$ ) and those who did not (Group B, $\mathrm{n}=46$ ) in terms of sex, duration of follow-up, or whether they underwent surgical intervention. The mean ages in the 2 groups, however, were significantly different (50.15 years in Group A vs 56.26 years in Group B, $\mathrm{p}=0.03$ ) (Table 1).

Operative treatments were performed in 38 cases, 9 (23\%) of which showed progression of OPLL at the final follow-up. Moreover, nonoperative conservative treatments were performed in 22 cases, 5 (22\%) of which showed progression of OPLL $(p=0.06)$. We analyzed the cases according to the operational approach used. An anterior approach was used in 14 cases, a posterior approach was used in 22 , and a combined approach was used in 2; these values were not significantly different among the groups. When the cases were analyzed according to the surgical method, we noted that a greater proportion of patients underwent nonfusion surgery in Group A than in Group B, although the difference was not significant (Table 2).

Kappa coefficients were used to measure interobserver (K1) and intraobserver (K2) agreement with respect to the the radiologically measured values, and both indicated high levels of reliability $(\mathrm{K} 1=0.82, \mathrm{~K} 2=0.80)$.
TABLE 1. Demographic and clinical characteristics of the patients

\begin{tabular}{lccc}
\hline \multicolumn{1}{c}{ Characteristic } & $\begin{array}{c}\text { Group A } \\
(\mathrm{n}=14)\end{array}$ & $\begin{array}{c}\text { Group B } \\
(\mathrm{n}=46)\end{array}$ & $\mathrm{p}$ Value \\
\hline Age in yrs (mean $\pm \mathrm{SD})$ & $50.15 \pm 8.5$ & $56.26 \pm 8.4$ & 0.03 \\
\hline Sex (M/F) & $12 / 2$ & $36 / 10$ & 0.71 \\
\hline $\begin{array}{l}\text { Follow-up in mos (mean } \\
\pm \text { SD) }\end{array}$ & $44.07 \pm 13.12$ & $25.19 \pm 8.11$ & 0.06 \\
\hline $\begin{array}{l}\text { Operation (yes/no) } \\
\text { No. of involved segments } \\
\text { (mean } \pm \text { SD) }\end{array}$ & $9 / 5$ & $29 / 17$ & 1.0 \\
\hline $\begin{array}{l}\text { VB connection (yes/no) } \\
\text { Trabeculation (yes/no) }\end{array}$ & $2 / 12$ & 3.6 & 0.002 \\
\hline VB = vertebral body. & $2 / 12$ & $43 / 3$ & $<0.001$ \\
\hline
\end{tabular}

The mean number of involved segments was significantly greater in Group A than in Group B (5.3 vs 3.6, p $=0.002$ ). Analysis of the ossification types showed that Group A had a higher proportion of cases with mixed types ( 3 cases of continuous, 10 of mixed, and 1 of local type) and Group B had a higher proportion of cases with segmental type (8 cases of continuous, 11 of mixed, 23 of segmental, and 4 of local type; $p=0.02$ ).

When analysis was performed according to the CT findings, there was a significant difference in the proportion of cases showing a connection between the OPLL mass and the vertebral body -2 of 14 cases in Group A versus 43 of 46 cases in Group B $(p<0.01)$. Moreover, the proportion of cases with trabeculation in the OPLL mass was significantly greater in Group B than in Group A (40 of 46 vs 2 of 14, p < 0.01).

In multivariate linear regression analysis $\left(\mathrm{r}^{2}=0.666\right)$, patient age $(\mathrm{p}=0.025)$, the number of involved segments $(\mathrm{p}=0.02)$, trabeculation in the OPLL mass $(\mathrm{p}=0.03)$, and connection between the OPLL mass and the vertebral body $(\mathrm{p}<0.001)$ showed significant relationships with the progression of the OPLL mass.

\section{Discussion}

Cervical OPLL, commonly found in middle-aged and elderly men, may initially present with minor symptoms, or patients may initially have no symptoms at all, but in some

TABLE 2. The progression of OPLL according to surgical approach and method

\begin{tabular}{lccc}
\hline Variable & Group A & Group B & p Value \\
\hline Approach & & & 0.361 \\
\hline Anterior & 2 & 12 & \\
\hline Posterior & 6 & 16 & \\
\hline Method & & & 0.431 \\
\hline Anterior + posterior & 1 & 1 & \\
\hline Fusion* & 3 & 14 & \\
\hline Nonfusiont & 6 & 15 & \\
\hline
\end{tabular}

* Includes anterior cervical discectomy and fusion, anterior cervical corpectomy and fusion, and posterior laminectomy and instrumented fusion. $\dagger$ Includes laminoplasty and laminectomy. 
patients, the disease slowly progresses, leading to the development of myelopathy. Conservative treatment options include bed rest, the application of a brace, and medication or local injection to relieve the symptoms. However, more invasive treatments (i.e., surgery) should be considered in cases of myelopathy, including gait disturbance or impairment of the fine movements of the hands. Understanding the natural history of OPLL is helpful in planning treatment options, especially in incidentally diagnosed cases involving patients with no symptoms. The natural history, however, is not clearly defined; thus, it is difficult to determine the correct course of treatment. Matsunaga et al. reported follow-up data for 450 patients over a period of at least 10 years, with a mean follow-up period of 17.6 years. ${ }^{9}$ Only $17 \%$ of the patients without myelopathy at the first visit developed myelopathy during the follow-up period. Based on Kaplan-Meier analysis, the estimated 30-year myelopathy-free rate was $71 \%$. Therefore, the authors proposed that OPLL patients with no myelopathic symptoms do not necessarily require preventive surgery. In contrast, $64 \%$ of patients who had symptoms of myelopathy in the early stages experienced aggravation of myelopathy. Through comparative analysis of cases in which patients presented with myelopathic symptoms versus cases in which patients presented with simple neck pain or neck pain and radiculopathy, Chang et al. evaluated the factors related to the manifestation of myelopathic symptoms in patients with OPLL. ${ }^{2}$ In patients with OPLL of the cervical spine, the manifestation of myelopathic symptoms was not related to the range of motion of the cervical spine or the number of involved segments. However, age at the time of diagnosis and the maximum compression of the spinal canal were related factors.

Chiba et al. analyzed the progression of OPLL by evaluating 131 OPLL patients who had undergone posterior decompression procedures, and progression was noted in $56.5 \%$ of patients at 2 years after surgery and was more frequent in continuous and mixed types of OPLL. ${ }^{3}$ Hori et al. evaluated patients with more than 5 years of followup after posterior decompression procedures. ${ }^{7}$ Progression was more common in cases of continuous and mixed types, especially among younger patients. However, these previous studies had some limitations; for example, the evaluations were performed only in patients who underwent operative treatments, and the measurements were performed using only simple radiography. Our findings were similar in that progression was more common in younger patients and mixed-type cases.

Previous studies reported that an OPLL mass was much more common in patients who had undergone operative treatment than in those who had only received conservative management. This may partly be due to biological stimulation after decompression, such as changes in the microcirculatory environment within the spinal canal. ${ }^{6,11}$ In the present study, there was no relationship between operative treatment and the progression of OPLL, and there was no difference between the operative method (fusion or no fusion) or the surgical approach (anterior or posterior). However, further studies analyzing progression based on the range of motion or combined instability by using dynamic radiography are required.
OPLL mainly develops through enchondral ossification, along with membranous ossification to some extent. ${ }^{5}$ McAfee et al. reported that OPLL is primarily composed of lamellar bone with mature haversian canals in addition to fibrous cartilage and woven bone in the calcified cartilages. ${ }^{10}$ That is, the posterior longitudinal ligament initially shows fibroblastic hyperplasia and collagen deposition, after which an immature ossification center is formed through gradual mineralization and cartilaginous ingrowth, thus eventually resulting in mature haversian canal formation and bone marrow development. ${ }^{4}$ Through CT analysis, we observed that the trabeculation developed within the OPLL mass during the last phase of OPLL progression. There was no progression in the cases with maturation of OPLL, wherein the characteristics were similar to those of normal cancellous bone. In addition, in the majority of cases where the OPLL mass was connected with the vertebral body, there was no additional progression. These findings should be useful in for predicting OPLL progression the clinical setting.

The limitations of this study include the relatively small number of cases and short follow-up period. However, previous reports show that OPLL progression spiked during the first 2 years and gradually increases for 5 years, indicating that our observation period, averaging 29.6 months, is sufficient for deriving a conclusion. Furthermore, the follow-up period was much longer in the OPLL progression group. Although multivariate linear regression analysis showed that the follow-up period was not related to the progression of the OPLL mass, it can be a confounding factor. However, this is a preliminary study, and further cases with longer follow-up periods will be analyzed prospectively to better determine the factors related to the disease progression.

\section{Conclusions}

The progression of OPLL was more common in younger patients with multilevel involvement and mixed-type OPLL masses. CT findings showing a connection of the vertebral body with the OPLL mass and trabeculation formation in the mass are useful for predicting nonprogression of the mass.

\section{References}

1. Chang H, Kong CG, Won HY, Kim JH, Park JB: Inter- and intra-observer variability of a cervical OPLL classification using reconstructed CT images. Clin Orthop Surg 2:8-12, 2010

2. Chang H, Song KJ, Kim HY, Choi BW: Factors related to the development of myelopathy in patients with cervical ossification of the posterior longitudinal ligament. J Bone Joint Surg Br 94:946-949, 2012

3. Chiba K, Yamamoto I, Hirabayashi H, Iwasaki M, Goto H, Yonenobu K, Toyama Y: Multicenter study investigating the postoperative progression of ossification of the posterior longitudinal ligament in the cervical spine: a new computerassisted measurement. J Neurosurg Spine 3:17-23, 2005

4. Epstein N: Diagnosis and surgical management of cervical ossification of the posterior longitudinal ligament. Spine $\mathbf{J}$ 2:436-449, 2002

5. Hashizume Y: Pathological studies on the ossification of 
the posterior longitudinal ligament (opll). Acta Pathol Jpn 30:255-273, 1980

6. Hirabayashi K, Miyakawa J, Satomi K, Maruyama T, Wakano K: Operative results and postoperative progression of ossification among patients with ossification of cervical posterior longitudinal ligament. Spine (Phila Pa 1976) 6:354-364, 1981

7. Hori T, Kawaguchi Y, Kimura T: How does the ossification area of the posterior longitudinal ligament thicken following cervical laminoplasty? Spine (Phila Pa 1976) 32:E551E556, 2007

8. Kawaguchi Y, Kanamori M, Ishihara H, Nakamura H, Sugimori K, Tsuji H, et al: Progression of ossification of the posterior longitudinal ligament following en bloc cervical laminoplasty. J Bone Joint Surg Am 83-A:1798-1802, 2001

9. Matsunaga S, Sakou T, Taketomi E, Komiya S: Clinical course of patients with ossification of the posterior longitudinal ligament: a minimum 10 -year cohort study. J Neurosurg 100 (3 Suppl Spine):245-248, 2004

10. McAfee PC, Regan JJ, Bohlman HH: Cervical cord compression from ossification of the posterior longitudinal ligament in non-orientals. J Bone Joint Surg Br 69:569-575, 1987

11. Takatsu T, Ishida Y, Suzuki K, Inoue H: Radiological study of cervical ossification of the posterior longitudinal ligament. J Spinal Disord 12:271-273, 1999
12. Tsuyama N: Ossification of the posterior longitudinal ligament of the spine. Clin Orthop Relat Res (184):71-84, 1984

\section{Author Contributions}

Conception and design: Choi, Chang. Acquisition of data: Choi, Baek. Analysis and interpretation of data: Choi. Drafting the article: Baek, Sheffler. Critically revising the article: Sheffler. Reviewed submitted version of manuscript: Choi, Sheffler. Approved the final version of the manuscript on behalf of all authors: Choi. Statistical analysis: Baek. Administrative/techni$\mathrm{cal} / \mathrm{material}$ support: Chang. Study supervision: Chang.

\section{Supplemental Information \\ Previous Presentation}

This article was presented in a podium section at the 41st annual meeting of the Cervical Spine Research Society in Los Angeles, California, December 5-7, 2013.

\section{Correspondence}

Byung Wan Choi, Department of Orthopedic Surgery, Inje University, Haeundae Paik Hospital, 1435, Jwa-dong, Haeundaegu, Busan, 612-030, Republic of Korea. email: alla1013@ naver. com. 